\title{
Structure Analysis of Magnetic Coupling Resonant for Wireless Power Transmission System
}

\author{
Qiang Zhao, Anna Wang, Hao Wang \\ Institute of Electric Power System and Motor Drives Northeastern, University, \\ Shenyang, China
}

\begin{abstract}
Using magnetically coupled resonators coils in a strongly coupled near field was suggested for wireless power transfer. A circuit model is presented along with a derivation of key system concepts such as frequency splitting, the maximum operating distance (critical coupling). Here, a simple circuit topological structure, the series-shunt-mixed resonant coupling, is presented with better performance in the transfer distance and efficiency. The theoretical analysis and experimental verifications of several resonant coupling structures have been investigated by several groups. In the experimental verification, a pair of resonant coils with $15 \mathrm{~cm}$ diameter was used. The experimental results show that high efficiency of $85 \%$ was achieved at a distance of $10 \mathrm{~cm}$ and $45 \%$ efficiency at $20 \mathrm{~cm}$. Through the developed model, it is seen that the system can transfer power over a midrange of a few meters and that impedance matching is important to achieve high efficiency.
\end{abstract}

Keywords-wireless power transfer; magnetic coupling resonant; frequency splitting; mixed-resonant circuit

\section{INTRODUCTION}

In recent years, there has been an increasing interest in the research and development of wireless power transfer (WPT) across large air gaps, which may finally eliminate the cable from mobile devices [1]. In this work, we extend prior analysis of coupled magnetic resonance to elucidate several key system concepts including: frequency splitting, critical coupling, and impedance matching. We present a model of magnetically coupled resonators in terms of an utilizable circuit structure [2], the serious-shunt mixed resonant circuits, was used for WPT. From the circuit analysis and numerical simulations, advantages of the series-shunt mixed-resonant coupling are demonstrated through comparisons with the traditional resonant-coupling schemes [3-5].

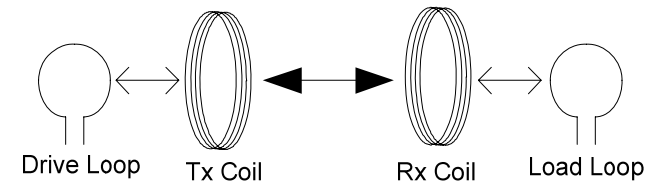

Figure 1. Magnetically coupled resonant wireless power system.

In previous research works [6-10], a physical distance was used to evaluate the performance of the power transfer system, while the size of the resonant coil was not taken into account. It was very easy to get a transfer efficiency of
$80 \%$ at a distance of $20 \mathrm{~cm}$ when the resonant coil diameter is $50 \mathrm{~cm}$. However, the transfer efficiency will become a big challenge if the coil diameter is reduced to $5 \mathrm{~cm}$, which is suitable to be embedded into small mobile devices such as cell-phones and cameras. Here, we propose a new concept of relative distance, which is defined as the ratio of distance to the resonant-coil diameter. In the following discussions, we will pay more attention on the high efficiency achieved in how many times of the coil diameter rather than in how many centimeters.

The paper is organized as follows. In the next section, we propose the model that we research in this paper, and some definitions and assumptions are given. In Section 3, the mixed-resonant coupling model based on functions is presented. In Section 4, the experimental verification is made to illustrate the efficiency of the algorithm. Finally, we conclude our paper in Section 5.

\section{MAGNETICALLY COUPLED RESONATORS MODEL}

Figure 1 shows a diagram of a resonant magnetically coupling system wherein a signal generator delivers power to load or device. Our experimental realization of the scheme consists of two self-resonant coils. One coil consists of a single turn drive loop and a multi-turn spiral coil, is coupled inductively to an oscillating circuit. When the RF amplifier powers the drive loop the resulting oscillating magnetic field excites the Tx coil which stores energy in the same manner as a discrete LC tank. The receive side functions in a similar manner, although a load replaces the power source, the $\mathrm{Rx}$ coil is coupled inductively to a resistive load. Self-resonant coils rely on the interplay between distributed inductance and distributed capacitance to achieve resonance. The coils are made of an electrically conducting wire of total length $l$ and cross-sectional radius $a$ wound into a helix of $n$ turns, radius $r$, and height $h$.

Figure 2 shows a equivalent circuit model of the wireless power transmission. Each of the four antenna elements are modeled as series resonators. Which are linked by mutual inductances via coupling coefficients $\mathrm{k} 12, \mathrm{k} 23$, k34. The exciting coils by the excitation source (highfrequency amplifier) VS and the single-turn coil. Loading coil is composed by a single-turn coil and the load. The transmitter coil and the receiver coil by having the same resonant frequency multi-turn coil. When the k12, k34 would be fixed, thus $\mathrm{k} 23$ is the remaining uncontrolled value which varies as a function of the distances between the transmitters to receiver. 


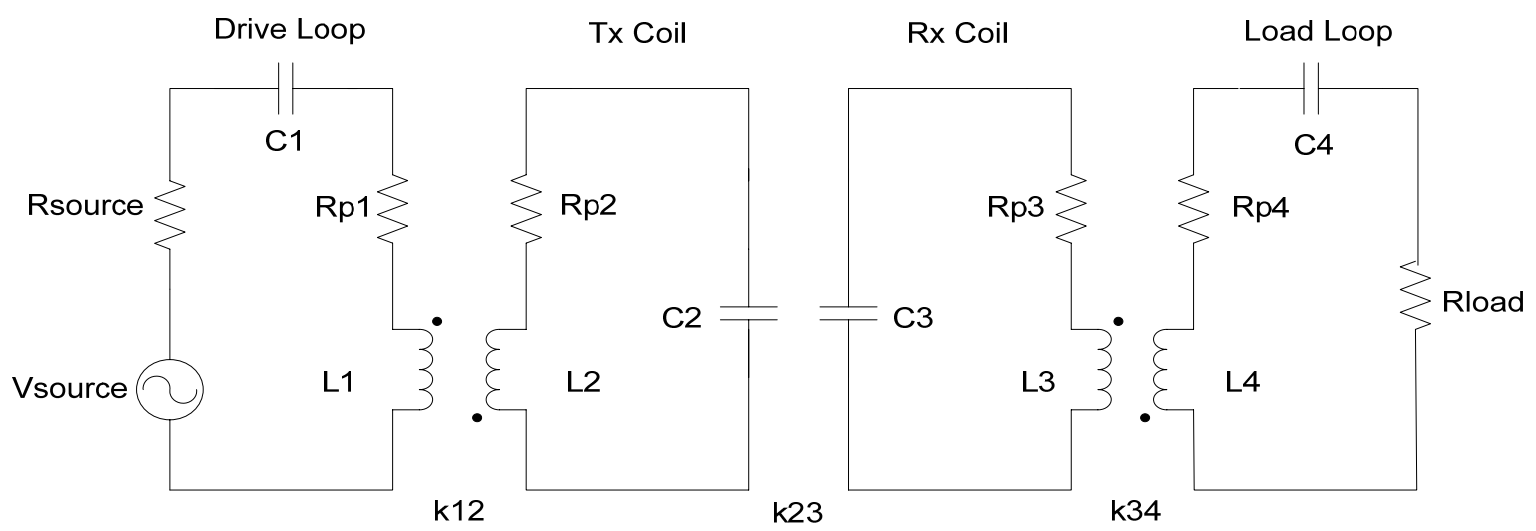

Figure 2. Equivalent circuit model of wireless power transmission.

\section{MIXED-RESONANT COUPLING MODEL}

The equivalent circuit model of the proposed resonant coupling is shown in Figure 3, whose five degenerated forms are the series-resonant circuit model, the parallelresonant circuit model, the series-parallel-resonant circuit model, the parallel-series-resonant circuit model and the mixed-resonant circuit model. Respectively capacitors $\mathrm{C} 1$ and $\mathrm{C} 4$ are in series and $\mathrm{C} 2$ and $\mathrm{C} 3$ are in shunt connected to the coils L1 and L2, which behave like an LC circuit to make the system resonant at a certain frequency so that the resonant coupling can be achieved.

The wireless power transmission system as the two port network with one port being input fed by the source and the other being output fed by the load. Based on the scattering
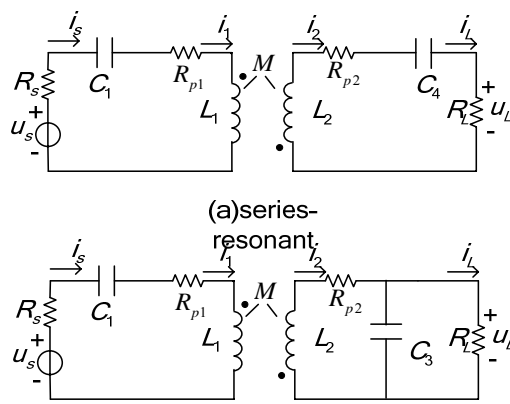

(c)series-parallel-

resonant
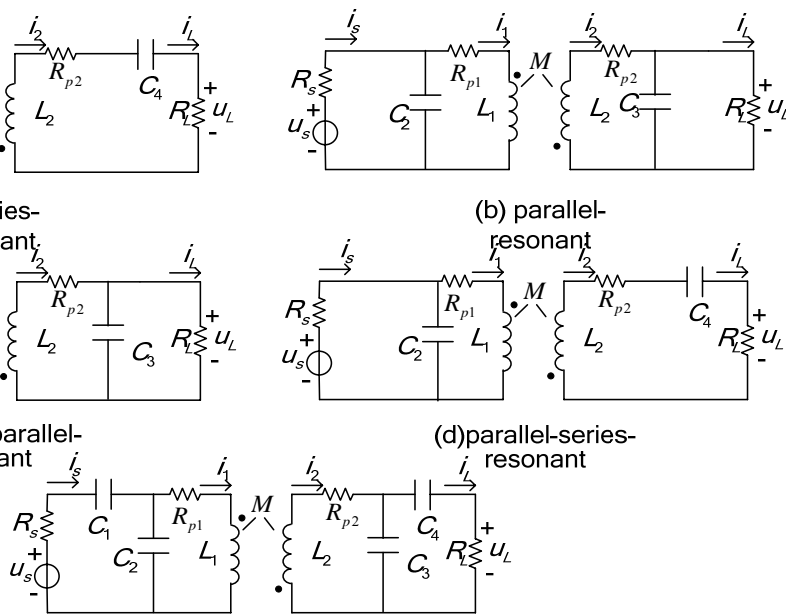

(b) parallel-

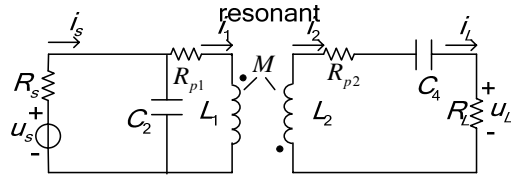

(d)parallel-seriesterms of the linear magnitude of the scattering parameter $\left|S_{21}\right|$, the function of power transfer efficiency is defined as $\eta=\left|S_{21}\right|^{2}$ when the network is matching at both ports, in which $\left|S_{21}\right|$ is given by

$$
S_{21}=2 \frac{V_{\text {Load }}}{V_{\text {Source }}}\left(\frac{R_{\text {Source }}}{R_{\text {Load }}}\right)^{1 / 2}
$$

For the above five models, we define the $S_{21}$ is a function of coefficient of mutual inductance $k$. So we get the substitution for the coupling circuit model as given in Eqs. (2)-(6).

(e)mixed-resonant

Figure 3. Equivalent circuit model of the proposed resonant coupling.

(a) Series-resonant circuit model:

$$
S_{21}=\frac{2 \cdot j \omega k \sqrt{L_{1} L_{2}} R_{L}}{\left(j \omega L_{1}+\frac{1+j \omega C_{1} R_{S}}{j \omega C_{1}}\right)\left(j \omega L_{2}+\frac{1+j \omega C_{4} R_{L}}{j \omega C_{4}}\right)+\omega^{2} k^{2} L_{1} L_{2}}
$$

(b) Parallel-resonant circuit model: 
(c) Series-parallel-resonant circuit model:

$$
S_{21}=\frac{2 \cdot j \omega k \sqrt{L_{1} L_{2}} \frac{1}{1+j \omega C_{2} R_{S}} \frac{1}{1+j \omega C_{3} R_{L}} R_{L}}{\left(j \omega L_{1}+\frac{R_{S}}{1+j \omega C_{2} R_{S}}\right)\left(j \omega L_{2}+\frac{R_{L}}{1+j \omega C_{3} R_{L}}\right)+\omega^{2} k^{2} L_{1} L_{2}}
$$

$$
S_{21}=\frac{2 \cdot j \omega k \sqrt{L_{1} L_{2}} \frac{1}{1+j \omega C_{3} R_{L}} R_{L}}{\left(j \omega L_{1}+\frac{1+j \omega C_{1} R_{S}}{j \omega C_{1}}\right)\left(j \omega L_{2}+\frac{R_{L}}{1+j \omega C_{3} R_{L}}\right)+\omega^{2} k^{2} L_{1} L_{2}}
$$

(d) Parallel-series-resonant circuit model:

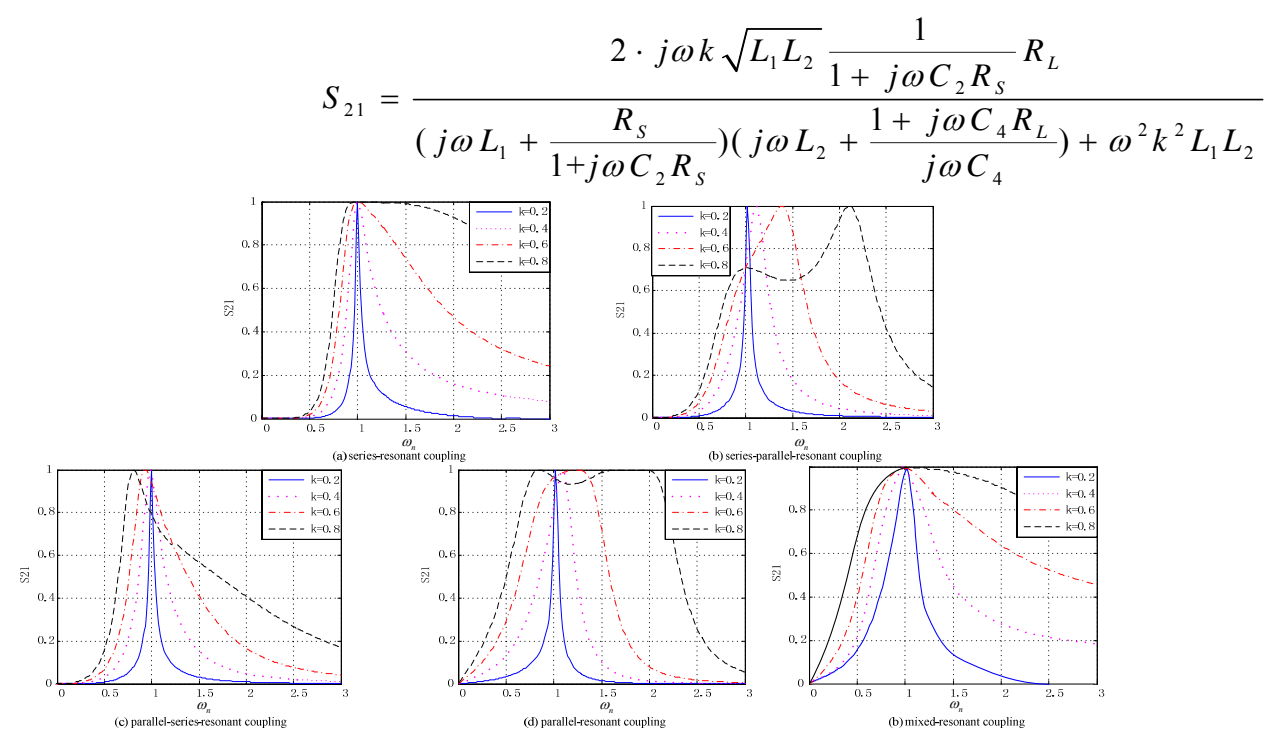

Figure 4. Curves of $\omega_{n}$ and efficiency at different.

(e) Mixed-resonant circuit model:

$$
S_{21}=\frac{2 \cdot j \omega k \sqrt{L_{1} L_{2}} \frac{j \omega C_{1}}{Z_{1}} \cdot \frac{j \omega C_{4}}{Z_{2}} R_{L}}{\left(j \omega L_{1}+\frac{1+j \omega C_{1} R_{S}}{Z_{1}}\right)\left(j \omega L_{2}+\frac{1+j \omega C_{4} R_{L}}{Z_{2}}\right)+\omega^{2} k^{2} L_{1} L_{2}}
$$

$$
\begin{aligned}
& Z_{1}=j \omega C_{1}+j \omega C_{2}-\omega^{2} C_{1} C_{2} R_{S} \\
& Z_{2}=j \omega C_{3}+j \omega C_{4}-\omega^{2} C_{3} C_{4} R_{L}
\end{aligned}
$$

Where

\section{MODEL VALIDATION AND EXPERIMENTAL SETUP OF A RESONANT COUPLED SYSTEM}

To demonstrate the superiority of the mixed-resonant coupling, a straightforward comparison among the different circuit models is conducted by simulation curve of $S_{21}$ magnitudes versus the coupling coefficient $k$ and $\omega_{n}$, as shown in Figure 4. The ratio of working frequency $\omega$ and the resonant frequency $\omega_{0}$ expressed as $\omega_{n}=\omega / \omega_{0}$ is the degree of deviation from the resonant frequency.

From the aforementioned analysis, it is obvious that the mixed-resonant coupling has a much better performance than others resonant couplings. Comparing Figure 4, we observe that the mixed-resonant coupling has inherited a longer effective transfer distance. The mixed-resonant coupling achieves much higher transfer efficiency than the shunt-resonant coupling because it inherits the low-sensitive feature from the series-resonant coupling. For the mixedresonant coupling, it is also noticed that a little sacrifice in the transfer efficiency will bring a longer effective transfer distance. This is because the mixed-resonant coupling is a mixture of the series- and shunt-resonant couplings. 


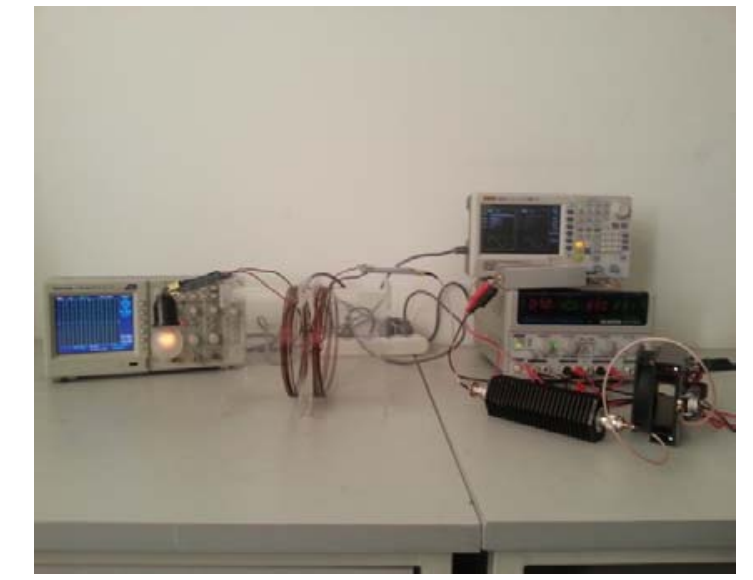

Figure 5. Experimental setup of the mixed-resonant coupling model.

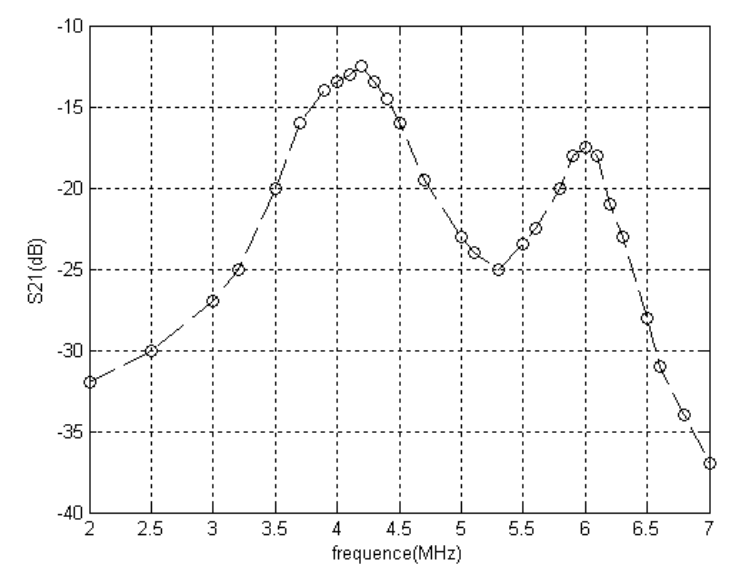

Figure 6. The $S_{21}$ of experimental in different frequencies.

The experimental setup used to validate the theoretical model is shown in Figure 5. There are two identical resonant coils winded by five turns tightly on the cylindrical Plexiglas, which are the transmitter (right) and the receiver (left). The detailed circuit parameters for the simulation model and the measurement are as follows: diameter of resonant coils is $15 \mathrm{~cm}$, a turn of the coil is 6 , and wire diameter is $2 \mathrm{~mm}, L=4.5 \mathrm{uH}, C=223 \mathrm{pF}, \mathrm{RL}=20 \Omega$, f0 $=$ 4.2 $\mathrm{MHz}$.

Transfer function $\left|S_{21}\right|$ near the resonant peak as measured experimentally and as calculated from the circuit model for the resonant coupled system with a single receiver is shown in Figure 6 . It shows a theoretical curve based upon the circuit model for a set of coupling coefficients that yields a good fit, along with experimental data points on a power decibel scale, however, we find that $S_{21}$ of the mixedresonant circuits with certain capacitance ratios

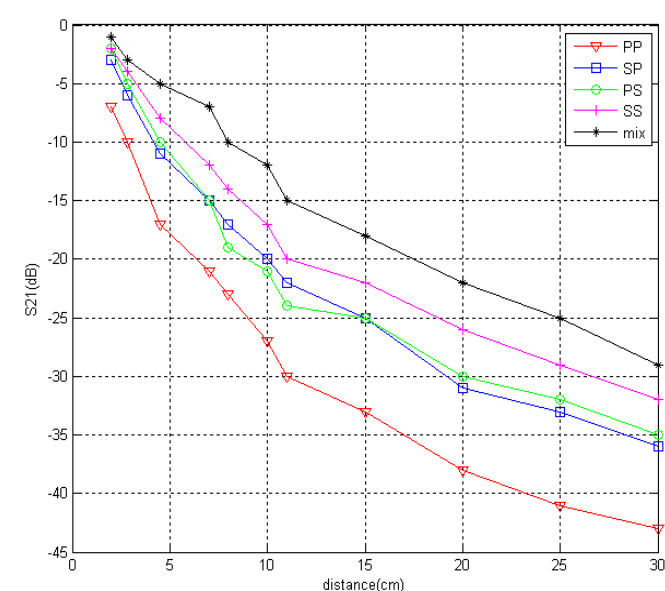

Figure 7. $S_{21}$ magnitudes versus distances for the SS, SP, PS, PP, and mixed structures.

According to the previous references, five kinds of circuits are studied for a more comprehensive analysis of other groups' contributions, including the seriescompensated primary and secondary (SS), seriescompensated primary and parallel-compensated secondary (SP), parallel-compensated primary and secondary (PP), parallel-compensated primary and series-compensated secondary (PS), and serious-shunt-mixed-compensated. Good agreements have been achieved between the simulation and experimental results.

The S-parameters versus distances of the SS, SP, PS, PP and mixed-resonant coupling circuits are measured and demonstrated in Figure 7, respectively.

From Figure 7 , we find that $S_{21}$ of all five circuit structures decreases dramatically with the increase of distances. It should be pointed out that the mixed structure is better than others structures with the chosen parameters, which can also be clearly seen in Figure 7. However, none of these circuits have shown good performance at long distance.

\section{CONCLUSIONS}

The work in this paper provides a deeper understanding of the underlying principles of coupled magnetic resonance, as well as a simple circuit model of the system. A derivation of the transfer function of this model reveals which concepts play a critical role in system performance: frequency splitting, operating range, and impedance matching. The proposed mixed-resonant coupling model has showed an excellent performance in WPT, making a further step for the magnetically coupled resonant structures toward practical applications. The other advantage of the model is the option between the effective transfer range and the maximum transfer efficiency, which allows WPT to be more adaptable in real applications. The previous approaches such as the series- and shunt-resonant coupling models do not have such abilities. 


\section{ACKNOWLEDGMENTS}

This work is partially supported by Shenyang Municipal Science and Technology Plan Projects Grant F13-053-2-00.

\section{REFERENCES}

[1] B. Cannon, J. Hoburg, D. Stancil, S. Goldstein, Magnetic resonant coupling as a potential means for wireless power transfer to multiple small receivers. IEEE Transactions on Power Electronics, 24(7), pp. 1819-1825, 2009.

[2] Linhui Chen, Shuo Liu, Yong Chun Zhou, Tie Jun Cui, An optimizable circuit structure for high-efficiency wireless power transfer. EEE Transactions on Industrial Electronics, 60(1), pp. 339-349, 2013.

[3] Y.-H. Kim, S.-Y. Kang, M.-L. Lee, B.-G. Yu, T. Zyung, Optimization of wireless power transmission through resonant coupling. In: Proceedings of CPE, pp. 426-431, 2009.

[4] F.Z. Shen, W.Z. Cui, J.T. Huangfu, L.X. Ran, Circuit analysis of wireless power transfer by "coupled magnetic resonance". In: Proceedings of IET CCWMC, pp. 602-605, 2009.

[5] C.K. Lee, W.X. Zhong and S.Y.R. Hui, "Effects of Magnetic Coupling of Non-adjacent Resonators on Wireless Power Domino-Resonator
Systems", IEEE Trans. Power Electronics, 27(4), pp. 1905-1916, 2012.

[6] Z. Pantic, B. Heacock, S.M. Lukic, Magnetic link optimization for wireless power transfer applications: Modeling and experimental validation for resonant tubular copper coils. In: IEEE Energy Conversion Congress and Exposition (ECCE), Raleigh, NC, September 2012.

[7] Shigeo Yakuno, Toshio Ishizaki, Ikuo Awai, Novel mobile wireless power transfer system based on coupled-lines and coupledline/resonator structures. In: KJMW2011 Digest, TH1-2-2, November 2011.

[8] A.P. Sample, D.A. Meyer, J.R. Smith, Analysis, experimental results, and range adaptation of magnetically coupled resonators for wireless power transfer. IEEE Transactions on Industrial Electronics, 58(2), pp. 544-554, February 2011.

[9] T. Imura, Y. Hori, Maximizing air gap and efficiency of magnetic resonant coupling for wireless power transfer using equivalent circuit and Neumann formula. IEEE Transactions on Industrial Electronics, 58(10), pp. 4746-4752, October 2011.

[10] J. Lee, S. Nam, Fundamental aspects of near-field coupling small antennas for wireless power transfer. In: IEEE Transactions on Antennas and Propagation, 58(11), pp. 3442-3449, November 2010. 